\title{
Molecular analysis of Trypanosoma cruzi isolates obtained from raccoons in Warren and Barren counties of Kentucky
}

\author{
LiPeng Bi ${ }^{1}$, Chad Groce ${ }^{2}$, Cheryl Davis ${ }^{1 *}$ \\ From UT-ORNL-KBRIN Bioinformatics Summit 2010 \\ Cadiz, KY, USA. 19-21 March 2010
}

\section{Background}

Trypanosoma cruzi, the etiologic agent of Chagas disease, infects a variety of wild mammals in the southern United States, but it has only recently been isolated from raccoons trapped in the state of Kentucky. The purpose of the present study was to use a molecular typing approach $[1,2]$, followed by DNA sequencing to determine the genotypes (type I, or types IIa-IIe) of 15 of the Kentucky isolates.

\section{Methods}

DNA samples were prepared from $15 \mathrm{~T}$. cruzi- isolates using a Qiagen mini kit, and PCR amplification was performed using published primers for the $24 \mathrm{~S}$ a rDNA sequence (D71 and D72), the non-transcribed spacer of the mini-exon genes (TC, TC1, and TC2), the $18 \mathrm{~S}$ rDNA sequence (V1 and V2), and TCZ1 and TCZ2 primers that amplify a 188-base pair segment of the repetitive 195-bp nuclear DNA sequence of T. cruzi. DNA sequencing (ABI 3130 Genetic Analyzer) was performed on all amplification products obtained from the PCR analysis of the RW2 and RB12 isolates (randomly selected to represent both Warren and Barren counties of Kentucky). The resulting sequences were edited before analysis using the BLAST database of the National Center for Biotechnology Information (NCBI) Genbank.

\section{Results}

All 15 isolates were positively confirmed as T. cruzi based upon PCR amplification of a195 bp repetitive genomic DNA sequence, and all 15 isolates showed identical PCR amplification results with all 4 sets of

\footnotetext{
* Correspondence: Cheryl.davis@wku.edu

${ }^{1}$ Department of Biology, Western Kentucky University, Bowling Green, KY 42101, USA
}

T. cruzi-specific primers. Two positive PCR samples were randomly selected for further DNA sequence analysis, and all samples were positively identified as the type IIa genotype of $T$. cruzi with max identities ranging from $94 \%-99 \%$.

\section{Conclusions}

The results of this study confirmed that all hemoculture isolates obtained from raccoons trapped in Warren and Barren counties of Kentucky are T. cruzi. Furthermore, all BLAST comparisons of amplicon DNA sequences showed high sequence identity to Type IIa strains of T. cruzi. The Type IIa strain of $T$. cruzi is the most commonly reported genotype reported from raccoons trapped in the U.S.A.

\section{Acknowledgements}

Author C. Davis gratefully acknowledges administrative support from $\mathrm{NIH}$ Grant Number 2 P20 RR-16481 from the National Center for Research Resources.

\section{Author details}

${ }^{1}$ Department of Biology, Western Kentucky University, Bowling Green, KY 42101, USA. ${ }^{2}$ College of Veterinary Medicine, Auburn University, Auburn, AL 36849, USA.

Published: 23 July 2010

\section{References}

1. Brisse S, Verhoef J, Tibayrenc M: Characterisation of large and small subunit rRNA and mini-exon genes further supports the distinction of six Trypanosoma cruzi lineages. Int J Parasitol 2001, 31:1218-1226.

2. Roeling D, Brown E, Barnabe C, Tibayrenc M, Steurer F, Yabsley M: Molecular typing of Trypanosoma cruzi isolates, United States. Emerg Infect Dis 2008, 14:1123-1125.

doi:10.1186/1471-2105-11-S4-P3

Cite this article as: Bi et al:: Molecular analysis of Trypanosoma cruzi isolates obtained from raccoons in Warren and Barren counties of Kentucky. BMC Bioinformatics 2010 11(Suppl 4):P3. 